\title{
Dynamic Mode Decomposition of Axisymmetric Synthetic Jet on the Limit of Its Formation
}

\author{
Tomáš Hyhlík \\ CTU in Prague, FME, Department of Fluid Dynamics and Thermodynamics \\ Technická 4, 16607 Prague, Czech Republic \\ tomas.hyhlik@fs.cvut.cz
}

\begin{abstract}
The modal decomposition of two flow fields related to axisymmetric synthetic jet creation has been done by using Dynamic Mode Decomposition (DMD). Both the case without synthetic jet formation defined by nondimensional stroke length $\mathrm{L}=0.615$ a the case with synthetic jet creation which is defined by $\mathrm{L}=1.174$ are studied. The analyses are based on the data by using ANSYS Fluent solver. Numerical results are validated by hot-wire anemometry data measured along the jet centerline. The vortex identification is based on residual vorticity, which allows identifying of the regions where continuum particle rotates. It is shown that in the case of $\mathrm{L}=1.174$ exist travelling vortex which diminishes two diameters behind orifice and this case is very close to the case of a synthetic jet without vortex roll-up. It is possible to expect that the vortex path would be shorter with decreasing stroke length until the vortex completely vanishes and the jet is not synthetized. The travelling vortex speed is measured by using vortex tracking and by using wavelength measured from DMD modes. It is shown that the vortex is travelling not only in the axial direction, but there is also a radial component which leads to the change of vortex ring size. The evaluation of travelling vortex speed leads to not typical behaviour because of a non-monotonous character of the vortex speed. It is shown that the vortex speed has an increasing-decreasing behaviour in the case of nondimensional stroke length $\mathrm{L}=1.174$.
\end{abstract}

Keywords: Synthetic Jet Flow, Vortex Ring, Residual Vorticity, Dynamic Mode Decomposition

\section{Introduction}

Synthetic jet creation is from the perspective of thermodynamics and fluid dynamics interesting because of its formation is connected with irreversibility. Positive entropy production can lead to vorticity flux from the orifice in the blowing part of the oscillation period. If there is enough vorticity flux, then vortex ring can be observed in the flow field. The created vortex ring is inducing velocity in the direction outside from the orifice. Synthetic jet can be generated only in the case where vortex ring induced velocity can overcome suction velocity. There are several typical behaviours of synthetic jet namely no synthetic jet case, synthetic jet without vortex roll-up and synthetic jet with vortex roll-up [1]. Concerning the existence of synthetic jet is probably the most interesting the case without vortex roll-up.

\section{Parameters of Synthetic Jets}

This section is describing most commonly used governing parameters of synthetic jet, see e.g. [1, 2]. Velocity scale is defined as time averaged blowing orifice centerline velocity over an entire cycle

$$
U_{0}=\frac{T}{T} \int_{0}^{T_{E}} u_{0}(r=0, t) \mathrm{d} t
$$

where $T_{E}$ is blowing time and $T=1 / f$ is oscillation period. Reynolds number can be determined using time averaged blowing orifice centerline velocity $U_{0}$ as

$$
\operatorname{Re}=\begin{gathered}
2 U_{0} D \\
v
\end{gathered}
$$

where $D$ is orifice diameter and $v$ is kinematic viscosity. Stokes number is defined as

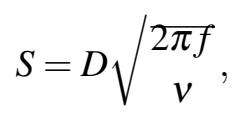

where $f$ is frequency. Stroke length of synthetic jet is length of fluid column that is pushed out during one cycle and can be calculated as

$$
L_{0}=U_{0} T .
$$




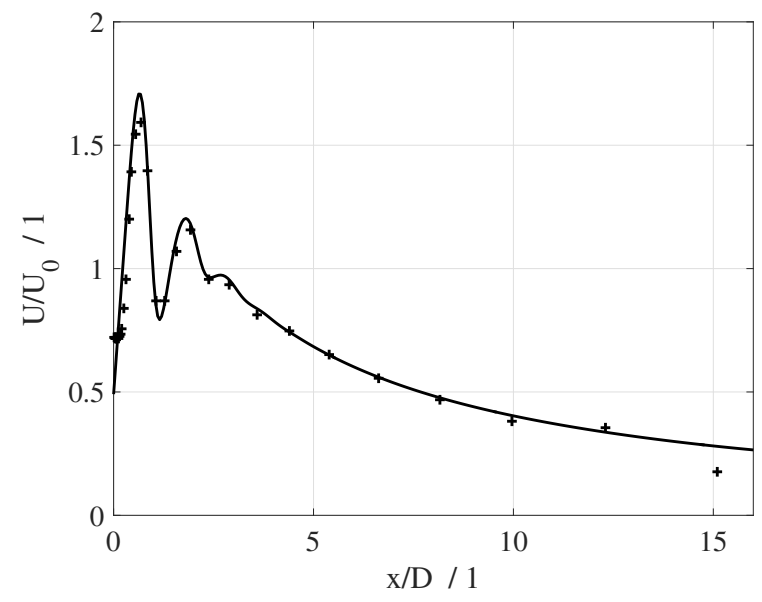

(a) Maximum volume

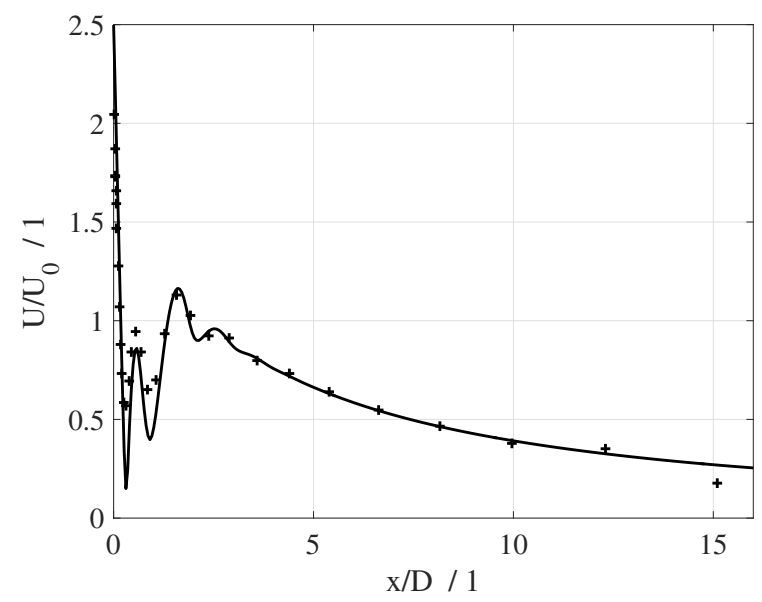

(c) Maximum suction

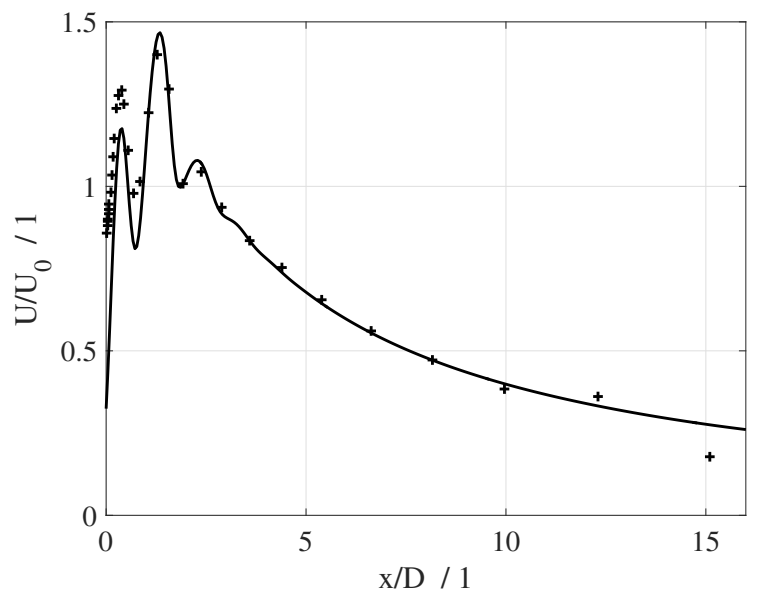

(b) Minimum volume

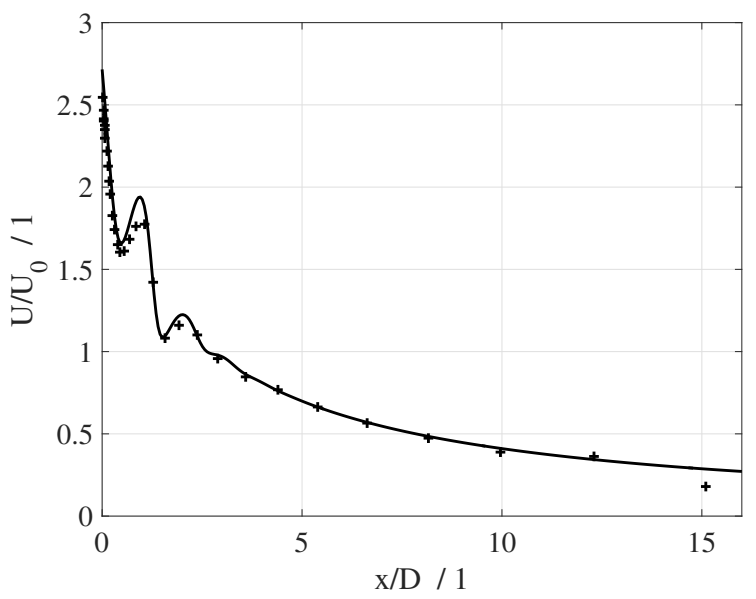

(d) Maximum blowing

Fig. 1: Instantaneous streamwise velocity at jet centerline for the case with $L=1.174$; solid line corresponds to numerical computation and dots are from hot-wire anemometry measurement [3].

Dimensionless stroke length is defined as

$$
L=\frac{L_{0}}{D}=\frac{R e}{S^{2}} \pi .
$$

The first studied regime corresponds to $L=0.615$, where $R e=76$ and $S=19.7$. The second studied regime is defined by $L=1.174$, where $R e=145$ and $S=19.7$. Formation criteria mentioned in reference [2] indicate an existence of synthetic jet in both cases, but the first case is practically on the limit of existence.

\section{Numerical simulation}

Unsteady incompressible laminar flow simulation has been performed using commercial solver ANSYS Fluent. The flow is assumed to be axisymmetric and is simulated on the computational mesh with 81550 cells by using non-iterative time advancement method with the second order implicit scheme. Fractional step scheme is used for pressure-velocity coupling. Convective terms are discretized using third order MUSCL scheme. Both the flow in the orifice and actuator cavity is included to get more accurate results in the simulation. The effect of the oscillating diaphragm on the left is replaced by velocity boundary condition which should guarantee achieving of required Reynolds number. The equation for inlet velocity is

$$
u(t)=\frac{\pi}{2} \frac{\operatorname{Re\eta }}{\rho D} \frac{D^{2}}{D_{L}^{2}} \sin (2 \pi f t),
$$




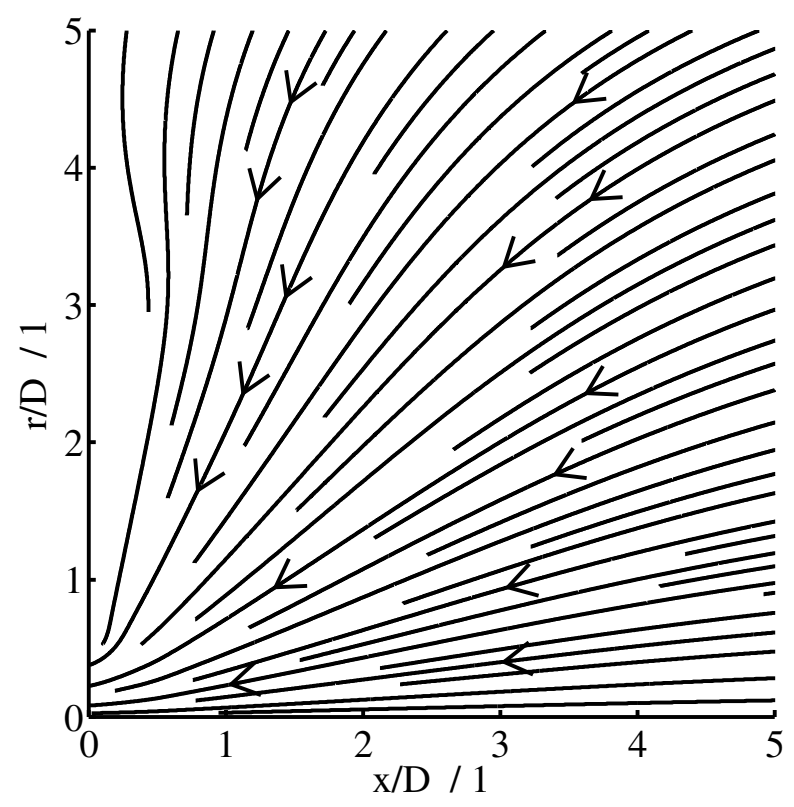

Fig. 2: Vector lines of imaginary part of first dynamic mode for $L=0.615$.

where $\eta$ is dynamic viscosity, $\rho$ is air density and $D_{L}$ is inlet diameter i.e. diameter of oscillating diaphragm.

The dimension of the computational domain was set according to the experimental measurement [3], where the jet is created in the cylindrical domain of $100 \mathrm{~mm}$ length and $60 \mathrm{~mm}$ in diameter, orifice diameter is $3.18 \mathrm{~mm}$.

Figure 1 shows instantaneous centerline streamwise velocity for four typical phases of synthetic jet work at $\mathrm{L}=1.174$ compared with hot-wire anemometry measurement [3]. The good agreement of computational data with measurement is observed. There are velocity peaks in Fig. 1 very close to the orifice which is placed at $x / D=0$. After velocity peak diminishes, the velocity is monotonously decreasing which indicate the creation of a jet flow. The existence of velocity peaks indicates some moving structure which diminishes close to the orifice.

\section{Dynamic Mode Decomposition}

Dynamic mode decomposition is a kind of modal decomposition method which was developed based on Koopman analysis of dynamical system [4]. DMD algorithm approximates Koopman modes from a finite set of data. Let the velocity field be represented by ensemble of snapshots sampling at interval $\Delta t$ with a form of matrix $\mathbf{V}_{1}^{N}=\left\{\mathbf{v}_{1}, \mathbf{v}_{2}, \ldots, \mathbf{v}_{N}\right\}$, where the column vector $\mathbf{v}_{j}$ contains velocity field at $j$ th time step. Let's consider linear mapping $\mathbf{A}$ that maps each snapshot to the next one in the following way

$$
\mathbf{v}_{j+1}=\mathbf{A} \mathbf{v}_{j}
$$

This mapping is assumed to be same over the full sampling interval $[0,(N-1) \Delta t]$. If the flow fields stem from a nonlinear process, this assumption corresponds to linear tangent approximation [5]. The assumption of a constant mapping between the snapshots $\mathbf{v}_{j}$ allows us to formulate the sequence of flow fields as a Krylov sequence

$$
\mathbf{V}_{1}^{N}=\left\{\mathbf{v}_{1}, \mathbf{A} \mathbf{v}_{1}, \mathbf{A}^{2} \mathbf{v}_{1}, \ldots, \mathbf{A}^{N-1} \mathbf{v}_{1}\right\}
$$

The goal of DMD is the extraction of dynamic characteristics such as eigenvalues and eigenvectors which are described by linear operator $\mathbf{A}$ based on sequence $\mathbf{V}_{1}^{N}$. Supposing that it is possible to write last snapshot $\mathbf{v}_{N}$ as linear combination of previous $N-1$ snapshots, for sufficiently long sequence of the snapshots

$$
\mathbf{v}_{N}=a_{1} \mathbf{v}_{1}+a_{2} \mathbf{v}_{2}+\ldots+a_{N-1} \mathbf{v}_{N-1}+\mathbf{r}
$$

where $\mathbf{a}^{T}=\left\{a_{1}, a_{2}, \ldots, a_{N-1}\right\}$ and $\mathbf{r}$ is residual vector.

Robustness is achieved by preprocessing step using singular value decomposition of data sequence [5]. The algorithm is working with a similar matrix to matrix A. Eigenvalues of similar matrix approximate eigenvalues of full mapping matrix A and provide temporal dynamics of the flow field. 


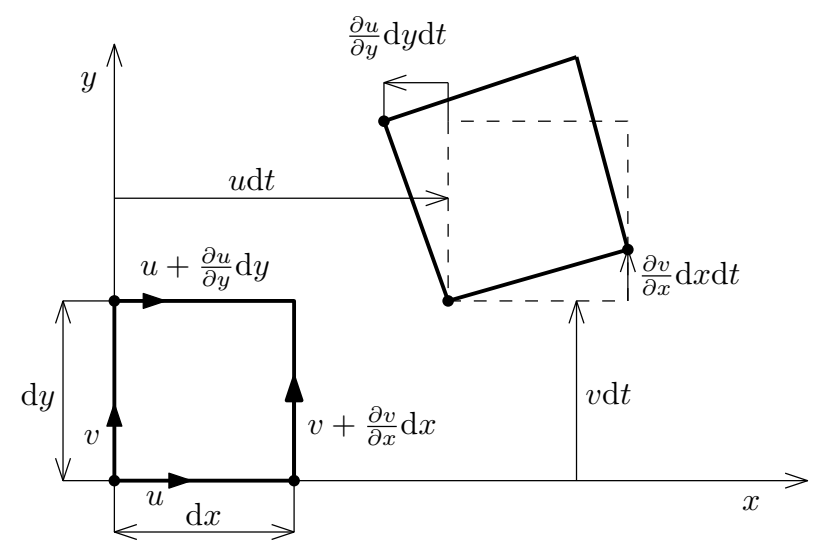

Fig. 3: Predominant rotation; the case where $\frac{\partial u}{\partial y}<0$ and $\frac{\partial v}{\partial x}>0$.

An example of a spatial structure of imaginary part of the first dynamic mode is depicted in Fig. 2 for the case of $L=$ 0.615. The velocity values are in the real part of the mode order of magnitude lover then in the imaginary part. The vector lines indicate the flow through orifice without the creation of any vortex structure. This structure indicates the case where the synthetic jet isn't created.

\section{Vortex Identification}

From the intuitive point of view, all of us believe we know what the vortex is. The one of the possible intuitive definitions can be: a vortex is a structure in the flow field which is characterised by the rotating motion of an infinite number of continuum particles around a common centre. This kind of definition is unclear because we are also expecting that the vortex is bounded finite structure. It is impossible to find the vortex boundary using the intuitive definition of the vortex.

The work [6] is offering an impressive concept of triple decomposition of motion where the tensor of velocity gradient is split into three parts. The first part is representing the elongation of a fluid portion; the second part is connected with rigid body rotation and the third part is the effective pure shearing tensor. The key idea of the mentioned work is to rotate the coordinate system to the so-called basic reference frame where it is possible to recognise clearly the kind of fluid motion.

It is possible to express the continuum particle speed $u_{i}$ when it changes its position from point $x_{j}$ to point $x_{j}+\mathrm{d} x_{j}$ as

$$
u_{i}\left(x_{j}+\mathrm{d} x_{j}\right)=u_{i}\left(x_{j}\right)+S_{i j} \mathrm{~d} x_{j}+\Omega_{i j} \mathrm{~d} x_{j},
$$

where $S_{i j}$ is symmetrical part of velocity gradient tensor

$$
S_{i j}=\frac{1}{2}\left(\frac{\partial u_{i}}{\partial x_{j}}+\frac{\partial u_{j}}{\partial x_{i}}\right)
$$

and $\Omega_{i j}$ is antisymmetric part of velocity gradient tensor.

$$
\Omega_{i j}=\frac{1}{2}\left(\frac{\partial u_{i}}{\partial x_{j}}-\frac{\partial u_{j}}{\partial x_{i}}\right)
$$

We have to find principal values and principal axes of the matrix of kind [7]

$$
\left(\begin{array}{ccc}
S_{x x} & S_{x y} & 0 \\
S_{y x} & S_{y y} & 0 \\
0 & 0 & S_{z z}
\end{array}\right),
$$

The value of $S_{z z} \neq 0$ is in general nonzero in the case of axisymmetric flow [7]. Eigenvalues of matrix (13) are

$$
\lambda_{1}=e_{1}+s, \lambda_{2}=e_{2}-s, \lambda_{3}=e_{3},
$$

where

$$
s=\frac{1}{2} \sqrt{\left(S_{x x}-S_{y y}\right)^{2}+4 S_{x y}^{2}}
$$




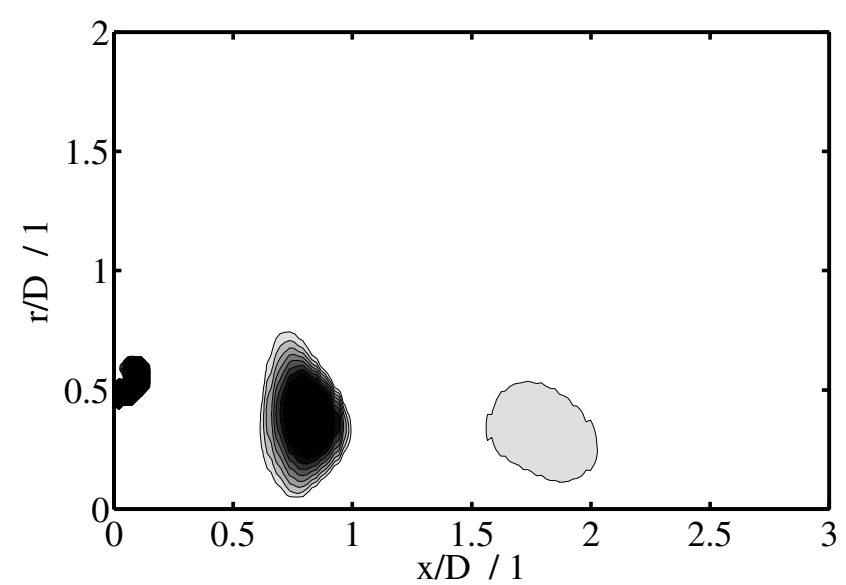

(a) $t / T=0.16444$

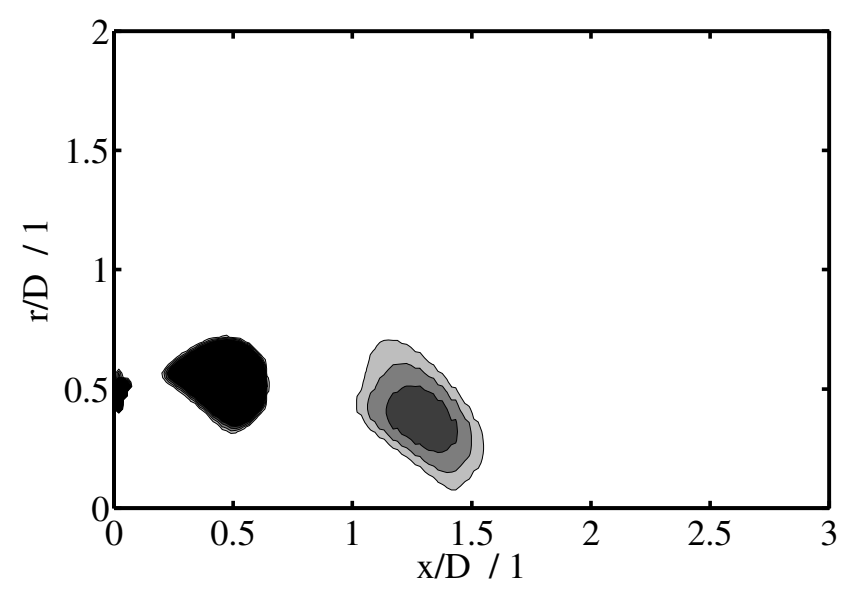

(c) $t / T=0.60889$
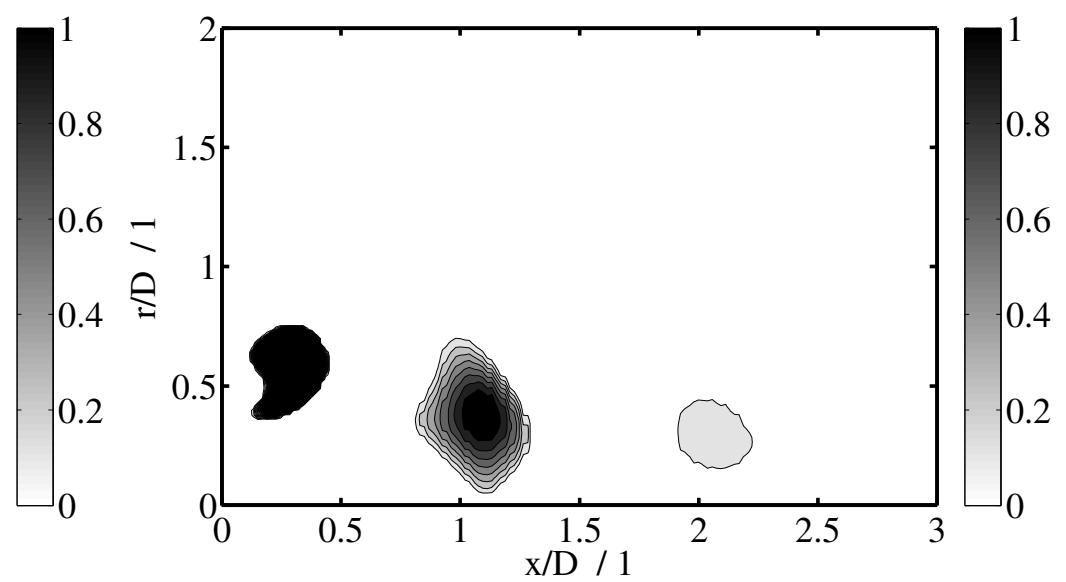

(b) $t / T=0.38667$
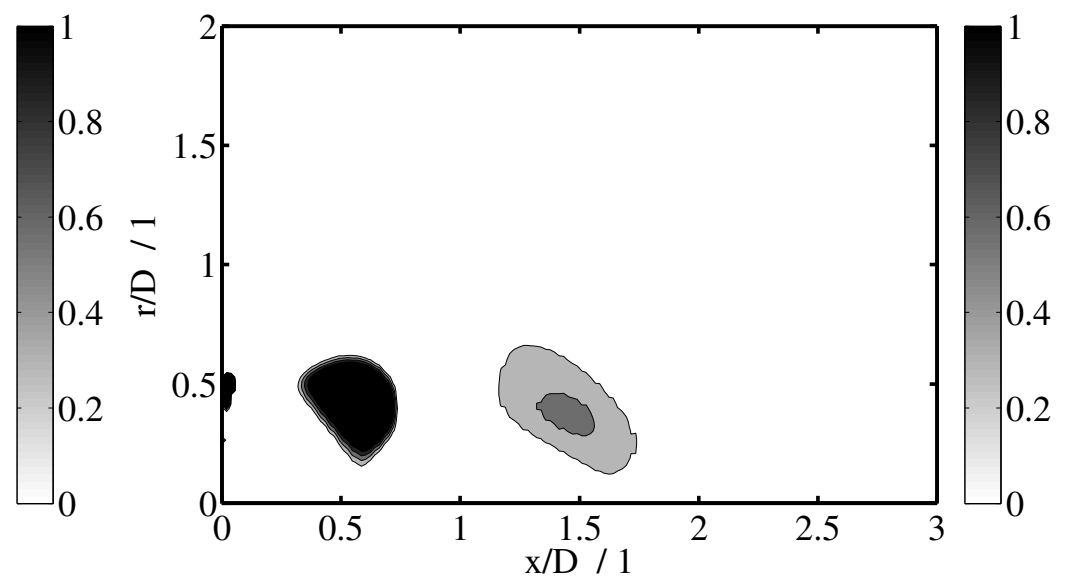

(d) $t / T=0.83111$

Fig. 4: Vortex identification by using residual vorticity at different phases for $L=1.174$.

and

$$
e_{1,2}=e=\frac{1}{2}\left(S_{x x}+S_{y y}\right), e_{3}=S_{z z}
$$

The velocity gradient tensor can be in principal axes expressed as

$$
\nabla \mathbf{v}=\left(\begin{array}{ccc}
e & 0 & 0 \\
0 & e & 0 \\
0 & 0 & e_{3}
\end{array}\right)+\left(\begin{array}{ccc}
s & 0 & 0 \\
0 & -s & 0 \\
0 & 0 & 0
\end{array}\right)+\left(\begin{array}{ccc}
0 & -\omega & 0 \\
\omega & 0 & 0 \\
0 & 0 & 0
\end{array}\right)
$$

where

$$
\omega=\frac{1}{2}\left(\frac{\partial v_{y}}{\partial x}-\frac{\partial v_{x}}{\partial y}\right)
$$

An arbitrary rotation around $z$ axis does not change the first and the third matrix in the relation (17). The first tensor in the relation (17) has no influence on shear deformation. It is possible to take into consideration only the second and the third part and rotate around $z$ axis about angle $\alpha=\frac{3}{4} \pi$

$$
\nabla \mathbf{v} \rightarrow \mathbf{D}\left(\begin{array}{ccc}
s & -\omega & 0 \\
\omega & -s & 0 \\
0 & 0 & 0
\end{array}\right) \mathbf{F}=\left(\begin{array}{ccc}
0 & s-\omega & 0 \\
s+\omega & 0 & 0 \\
0 & 0 & 0
\end{array}\right) .
$$




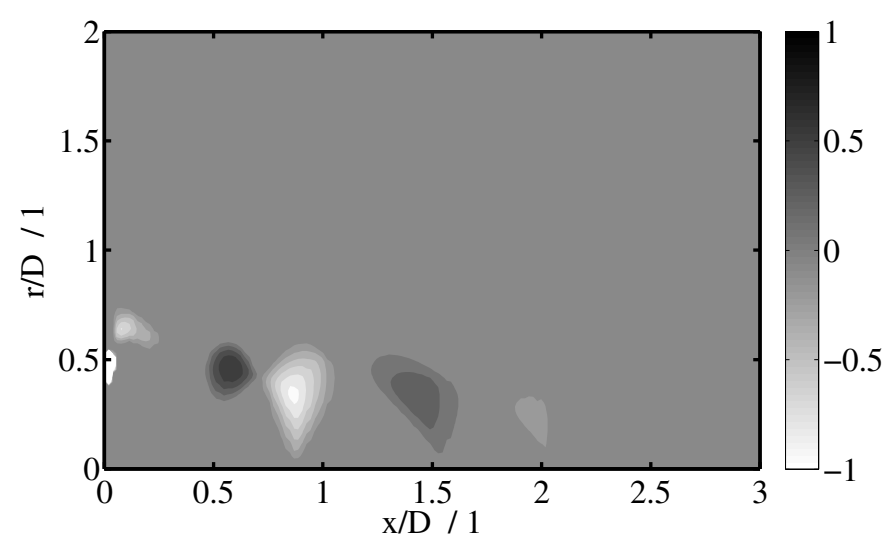

(a) Imaginary part of first DMD mode; $f_{1}=f$

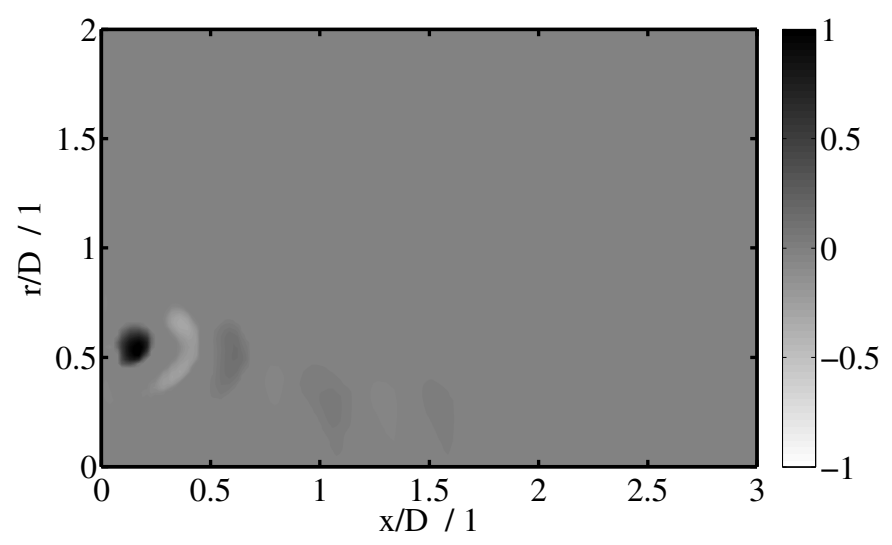

(c) Imaginary part of second DMD mode; $f_{2}=2 f$

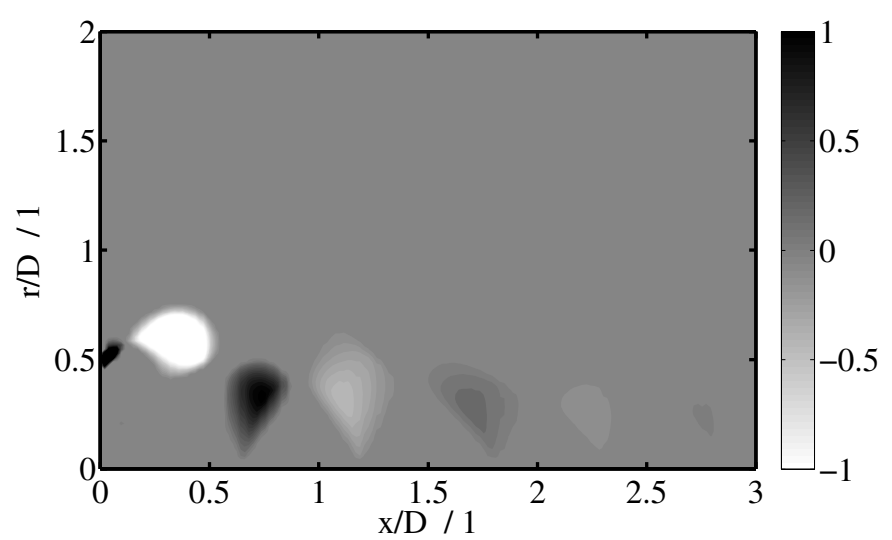

(b) Real part of first DMD mode; $f_{1}=f$

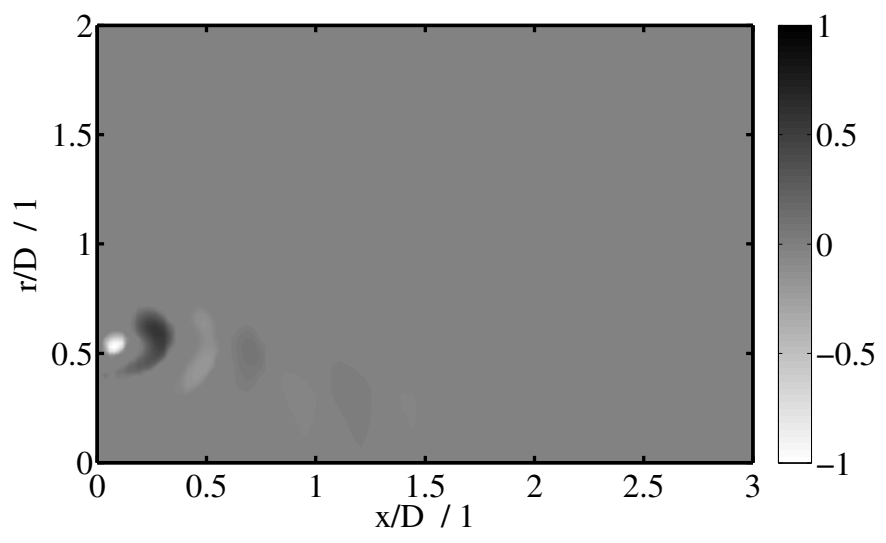

(d) Real part of second DMD mode; $f_{2}=2 f$

Fig. 5: Residual vorticity contours of DMD modes for $L=1.174$.

Velocity gradient tensor is after transformation in so called basic reference frame [6]. It is possible to examine kinematics of the flow in the basic reference frame clearly.

All of us intuitively know that vortex is somehow connected with vorticity but on the other hand, we are aware that there are cases like laminar shear flow where the value of vorticity is nonzero, and there is not any vortex. The work [6] defines vortex as the case where the value of vorticity predominates the shear $|\omega| \geq|s|$. Let's assume only shear and vorticity parts of the velocity gradient tensor, and then it has only two nonzero components in the basic reference frame $\frac{\partial u}{\partial y}$ and $\frac{\partial v}{\partial x}$ having opposite sign in the case of predominant vorticity, see figure 3 . The residual shear parameter is in this case $s_{R E S}=0$ and shear parameter is $s_{S H}=s$. Let the residual vorticity be

$$
\omega_{R E S}=\operatorname{sgn}(\omega)[|\omega|-|s|] .
$$

and shear vorticity be

$$
\omega_{S H}=\operatorname{sgn}(\omega)|s| .
$$

Fig. 4 shows travelling vortexes in different phases for the case of $L=1.174$ which are identified by using residual vorticity. The possibility to use residual vorticity to identify the structure of DMD modes is documented in Fig. 5.

\section{Results}

There are three types of behaviour connected with spatial DMD modes. The first one is the case where the real part dominates over imaginary one. This kind of behaviour in the dynamic mode can indicate travelling wave-like structure in the flow field [8]. This behaviour is not present in the case of synthetic jet flow. 


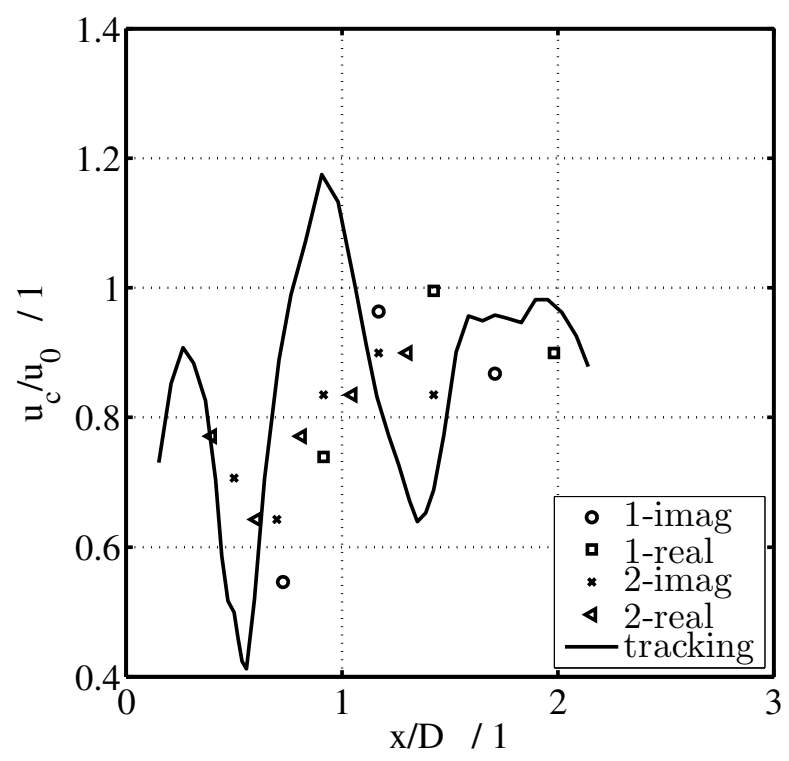

Fig. 6: Vortex speed normalized by averaged blowing orifice centerline velocity.

The second kind of behaviour occurs in the case where the imaginary part of dynamic mode dominates over the real one. This can be observed in the case where the synthetic jet isn't created, i.e. $L=0.615$. The most dominant mode is, in this case, the first dynamic mode where imaginary part is an order of magnitude greater than the real one. The spatial structure of this mode is depicted in Fig. 2. The structure documents the case where the air is cyclically sucked and blown from and to the whole domain through the orifice without any synthetic jet creation.

The third kind of behaviour where the real and imaginary parts are similar, but there is space shift in the flow field. The space shift is typically one-quarter of a wavelength which can be measured by using spatial structure of the modes. This kind of behaviour is typical for the travelling vortex structures and can be observed in Fig. 5. There is a travelling vortex ring in the flow field depicted in the mentioned figure. The vortex ring is travelling from left to right in the $x$ direction as documented in Fig. 4. It is possible to observe opposite vortex rotation in the DMD modes in the figure, where vortices are visualised using residual vorticity. The medium grey represents the region without any vortex. The area in which the colour is lighter than medium grey is connected with one direction of rotation and the area where is the colour darker than medium grey is related to opposite rotation. It is possible to measure wavelength directly in the case of every single DMD mode. The wavelength is the distance of two closest local extrema and is changing with spatial position. The vortex speed is connected with wavelength as

$$
u_{c}=\lambda f_{m},
$$

where $f_{m}$ is frequency connected with given mode.

Travelling vortex speed is measured both by using vortex tracking and by using wavelength measured from the DMD modes in Fig. 5. Vortex tracking is based on the evaluation of the vortex position by using residual vorticity contours as shown in Fig. 4. The vortex speed is depicted in Fig. 6. The observed behaviour is not the typical one [9]. It is possible to observe that the vortex is travelling not only in the axial direction, but there is also a radial component which leads to the change of vortex ring size, see Fig. 4. The evaluation of travelling vortex speed leads to not typical behaviour because of a non-monotonous character of the vortex speed in Fig. 6. It is visible that the vortex speed has an increasing-decreasing behaviour. There are peaks in the vortex speed distribution, but this behaviour is observed next to the orifice where is the vortex created. The vortex speed is affected by some error due to the size of the area where the vortex exists and due to the size of the vortex. Vortex speed evaluation is based on the data which are manually read from the figures.

\section{Conclusions}

The numerical simulations of two regimes of synthetic jet work have been performed by using ANSYS Fluent code. Obtained results are compared with hot-wire anemometry measurement. The good agreement of computational data with measured is observed. The focus is concentrated on the study of obtained unsteady flow fields by using dynamic mode decomposition and on the identification of vortex structures using residual vorticity. The three possible behaviours which are connected with DMD modes are discussed. It has been shown that the case without the existence of synthetic jet is joined with a predominance of the imaginary part of the first DMD mode. The typical spatial structure of DMD modes is shown 
in the case of travelling vortex where the vortexes are identified using residual vorticity. The vortex structure identification based on residual vorticity is modified to apply to axisymmetric flow. The speed of travelling vortex ring is evaluated by using vortex tracking and by using DMD modes, where the vortices are identified using residual vorticity. The contours of residual vorticity of individual modes are used to measure a spatial change of wavelength which together with the mode frequency allows the calculating speed of travelling vortex.

\section{Acknowledgments}

The support from Czech Science Foundation under Grant No. 14-08888S is gratefully acknowledged. I am grateful to Dr. J. Kordík of Institute of Thermomechanics AS CR for sharing his data from hot-wire anemometry measurement.

\section{References}

[1] Z. Trávníček, Z. Broučková, J. Kordík, "Formation Criterion for Axisymmetric Synthetic Jets at High Stokes Numbers," AIAA Journal, vol. 50, no. 9, pp. 2012-2017, 2012.

[2] R. Holman, Y. Utturkar, R. Mittal, B. L. Smith, L. Cattafesta, "Formation Criterion for Synthetic Jets," AIAA Journal, vol. 43, no. 10, pp. 2110-2116, 2005.

[3] Z. Trávníček, Z. Broučková, J. Kordík, T. Vít, "Visualization of Synthetic Jet Formation in Air," Journal of Visualization, vol.18, no. 4, pp. 595-609, 2015.

[4] C. W. Rowley, I. Mezić, S. Bagheri, P. Schlatter, D. S. Henningson, "Spectral Analysis of Nonlinear Flows," J. Fluid Mech., vol.641, pp. 115-127, 2009.

[5] P. J. Schmid, "Dynamic Mode Decomposition of Numerical and Experimental Data," J. Fluid Mech., vol.656, pp. 5-28, 2010.

[6] V. Kolář, "Vortex Identification: New Requirements and Limitations," International Journal of Heat and Fluid Flow, vol.28, pp. 638-652, 2007.

[7] T. Hyhlík, "Method of Vortex Structure Identification in Axisymmetric Flow Field," Manufacturing Technology, vol.15, no. 5, pp. 845-850, 2015.

[8] O. Frederich, D. M. Luchtenburg, "Modal Analysis of Complex Turbulent Flow," Seventh International Symposium on Turbulence and Shear Flow Phenomena, Canada, Ottawa, 2011.

[9] B. L. Smith, A. Glezer, "The Formation and Evolution of Synthetic Jets," Physics of Fluids, vol. 10, no. 9, pp. 2281-2297, 1998. 